\title{
THE
}

\section{Two-Element Dielectric Antenna Serially Excited by Optical Wavelength Multiplexing}

\author{
Everett E. Crisman \\ University of Rhode Island \\ John S. Derov \\ P. H. Carr \\ S. D. Mittleman \\ D. D. W. Liu
}

Follow this and additional works at: https://digitalcommons.uri.edu/che_facpubs

Part of the Chemical Engineering Commons

Terms of Use

All rights reserved under copyright.

\section{Citation/Publisher Attribution}

Crisman, E. E., Derov, J. S., Carr, P. H., Mittleman, S. D., \& Liu, D. D.-W. (1999). Optoelectronics - TwoElement Dielectric Antenna Serially Excited by Optical Wavelength Multiplexing. Optics Letters, 24(4), 235-237. doi: 10.1364/OL.24.000235

Available at: http://dx.doi.org/10.1364/OL.24.000235

This Article is brought to you for free and open access by the Chemical Engineering at DigitalCommons@URI. It has been accepted for inclusion in Chemical Engineering Faculty Publications by an authorized administrator of DigitalCommons@URI. For more information, please contact digitalcommons-group@uri.edu. 


\title{
Two-element dielectric antenna serially excited by optical wavelength multiplexing
}

\author{
E. E. Crisman, ${ }^{*}$ J. S. Derov, P. H. Carr, and S. D. Mittleman \\ Electromagnetics Technology Division (AFRL/SNHA), Air Force Research Laboratory, Hanscom Air Force Base, Massachusetts 01731-3010 \\ D. D.-W. Liu \\ Advanced Technology Division, Sanders-A Lockheed Martin Company, P.O. Box 868, MER 24-1583, Nashua, New Hampshire 03061
}

Received October 1, 1998

\begin{abstract}
A single pulsed laser beam containing multiple wavelengths (wavelength multiplexing) is employed to activate two semiconductor antennas in series. The dielectric nature of the semiconductors permits serial cascading of the antenna elements. Recently observed nonlinear characteristics of the radiated field as a function of the free carrier accelerating (bias) voltage are used to minimize the small interactions between elements. We demonstrate that the temporal electromagnetic radiation distribution of two serial antennas is sensitive to the three-dimensional pattern of the optical excitation source. One can, in turn, vary this distribution continuously by optical means to reconfigure the array. (c) 1999 Optical Society of America

OCIS codes: $160.5140,260.5150,140.0140,060.4230,350.4010$.
\end{abstract}

In this Letter we report the performance of two photoconductive antenna elements excited in series by picosecond laser pulses. Rather than provide a separate optical pathway to each element, we entrain two wavelengths in a single optical beam. This approach results in a more compact antenna array. Each semiconductor element is tailored to respond to only one of the wavelengths. Such an array can be reconfigured by the wavelength content of the optical beam. When this array is combined with coplanar excitations (multiple excitations of the same element), threedimensional electromagnetic (EM) source antennas are readily achieved.

Because of their large bandwidth, pulsed microwave sources operating in the gigahertz range have been suggested for a number of applications, such as groundpenetrating radar (antipersonnel mine identification, utilities location), remote triggering encoders in mining, automobile anticollision systems, aircraft type identification, and portable medical and security scanning. ${ }^{1}$ The use of semiconductor radiating elements combined with diode lasers and laptop-sized computers should result in compact, portable units compared with systems in use today. ${ }^{2}$ Such configurations do not require the microwave plumbing and the mechanical steering associated with conventional centimeterwavelength sources and antennae.

Lasers (operating in either the $\mathrm{cw}$ or the pulse mode) activate the semicoductor elements by generating free carriers. ${ }^{3-6}$ Those photocarriers are accelerated in an externally applied electric field to produce EM radiation. When they are not illuminated, the semiconductor elements are essentially transparent to high-frequency EM fields and therefore are not interactive with other components. Because of their dielectric nature, inactive semiconductor elements also have a lower microwave cross section then their metallic counterparts.

The concept and the general experimental setup of the laser-induced pulsed picosecond EM source have been described in detail elsewhere. ${ }^{5}$ Briefly, a modelocked $Q$-switched YLF laser system provides $50-\mu \mathrm{J}$ pulse energy with 80-ps pulse duration at a wavelength of $1053 \mathrm{~nm}$. The laser pulses are selectively chosen by use of a Pockels cell at a repetition rate of $378 \mathrm{~Hz}$, and a KDP frequency doubler converts the pulses to a wavelength of $527 \mathrm{~nm}$. The doubling is accomplished in such a manner as a to allow a significant amount of energy to radiate at $1053 \mathrm{~nm}$ as well. A photodetector is used to trigger a TEK11802 sampling scope. Pulses of 100-ps duration are readily resolved, and 10 -ps resolution is possible with this setup. A $20-\mathrm{kV}$ $5-\mu \mathrm{s}$ bias pulse is synchronized with the laser pulse. Using pulsed bias instead of dc bias reduces heating and surface flashover on the elements. Figure 1 illustrates a serial configuration of the photoconductive array of two semiconductor elements photoactivated by such a dual-wavelength laser source.

The two-element system consists of a GaAs wafer in the rear and an InP wafer in front. Undoped GaAs (bandgap $E_{g}=1.43 \mathrm{eV}$ at room temperature) strongly absorbs the 526-nm wavelength but is transparent at the 1053-nm wavelength, whereas the InP with Fe impurities $\left(E_{g}=1.32 \mathrm{eV}\right.$ at room temperature)

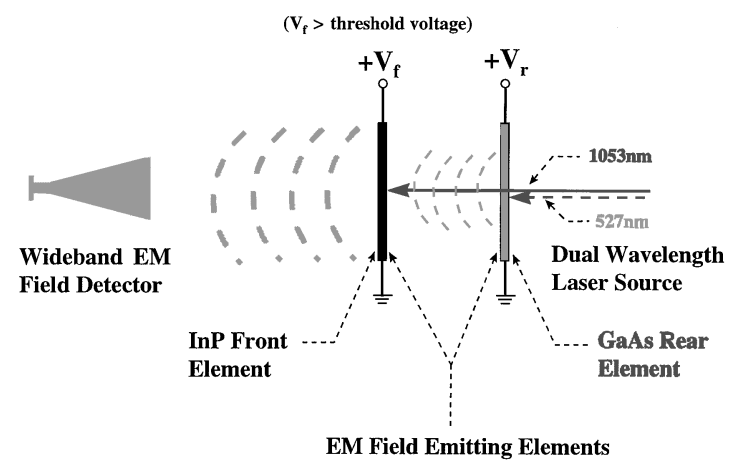

Fig. 1. Laser-induced phased picosecond EM source series configuration for two-color laser source exciting two EM radiating elements. 
absorbs strongly at $1053 \mathrm{~nm}$. As a result, in-line array elements are activated. For optimization, one can adjust the absorption edge and thus the wavelength sensitivities of the semiconductors by varying the density and species of the impurities.

The nonlinear characteristics of the radiated field versus the bias field generated by a gigahertz photoconducting antenna have been reported elsewhere. ${ }^{7}$ The amplitude of such fields, $E(t)$, is proportional to their final velocity $(v)$ :

$$
E(t) \propto \frac{e \nu(1-R)}{\hbar \omega} \int_{-\infty}^{t} \mathrm{~d} t^{\prime} I_{\mathrm{op}}\left(t^{\prime}\right) \exp \left[\frac{\left(t-t^{\prime}\right)}{\tau_{r}}\right],
$$

where $e$ is the unit charge quantity, $v$ is the carrier velocity, $R$ is the optical reflectivity of the semiconductor at frequency $\omega, e \nu$ is the photon energy, $I_{\mathrm{op}}$ is the optical intensity, and $\tau_{r}$ is the photocarrier lifetime.

Typically the accelerating carriers (usually electrons) in the semiconductor can reach their final velocity within a few picoseconds or less, i.e., a time much shorter than the optical pulse duration of $\sim 80$ ps. Consequently the waveform of the generated microwave pulse emulates the profile of the optical pulse in the time domain. The carrier velocity is not linear with the bias electric field, and some threshold value exists above which a saturation plateau is observed. $^{7}$ This relationship is shown in Fig. 2.

When the bias field is set at the plateau, the photoinduced EM signal will become insensitive to variations of the bias field. Taking advantage of this property, we established the bias field of the front element above the threshold value for $\operatorname{InP}(6 \mathrm{kV} / \mathrm{cm}$ for GaAs and $12 \mathrm{kV} / \mathrm{cm}$ for $\mathrm{InP}$ ). Therefore the EM field arriving from the rear ( $\mathrm{GaAs})$ element does not affect the generation of the EM field in the front (InP) element (see Fig. 3). Furthermore, the EM pulse and the optical pulse will arrive essentially simultaneously at the front element, thus ensuring that the combined (front and rear elements) EM field will be in phase. In this configuration the couplings between the elements are expected to be minimal, and the total radiation signal in the far field is given as the superposition of the individual EM fields. Higher gain and beam-width narrowing of the far-field EM radiation are thus expected. ${ }^{8,9}$ These expectations were confirmed experimentally, as shown in Fig. 4. Note that in Fig. 4 the pattern from the combined elements approaches the cosine squared shape, whereas the singleelement pattern is significantly broader. This finding is consistent with dipole versus monopole behavior. The final velocity of free carriers in III-V semiconductors decreases with electric field above some threshold. ${ }^{10}$ Such negative differential conductivity behavior is applicable to photocarriers as well ${ }^{11}$ and can be used to further reduce interelement coupling. By taking advantage of the fact that the microwave signal has the opposite polarity from the bias field, ${ }^{12}$ we can ensure that the electromagnetic pulse from the rear element, when it arrives at the front element, will decrease the bias field there. Referring to Fig. 3, if the bias on the front element is to the right of the plateau, decreasing the bias will actually increase the carrier velocity. Note, finally, that the direction of the far-field maximum amplitude aligns with the optical beam direction, permitting the EM pulse to be steered optically.

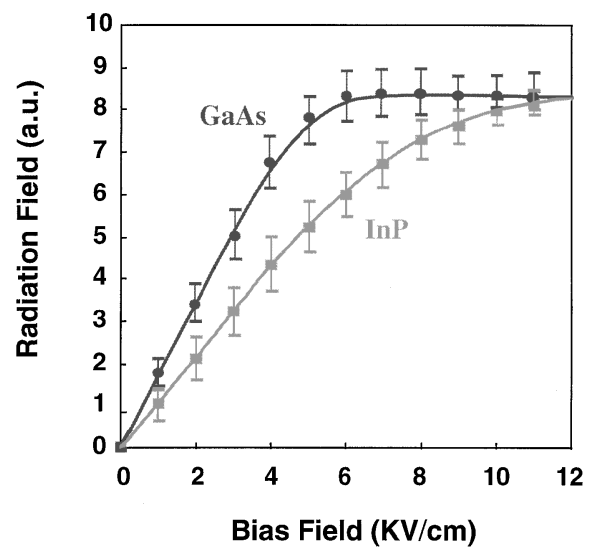

Fig. 2. EM field amplitude as a function of the bias field for $\mathrm{GaAs}$ and $\mathrm{InP}$ with the optical fluence at $0.3 \mathrm{~J} / \mathrm{cm}^{2}$. Two curves are scaled to have a same radiation field magnitude at the bias field of $12 \mathrm{kV} / \mathrm{cm}^{7}$

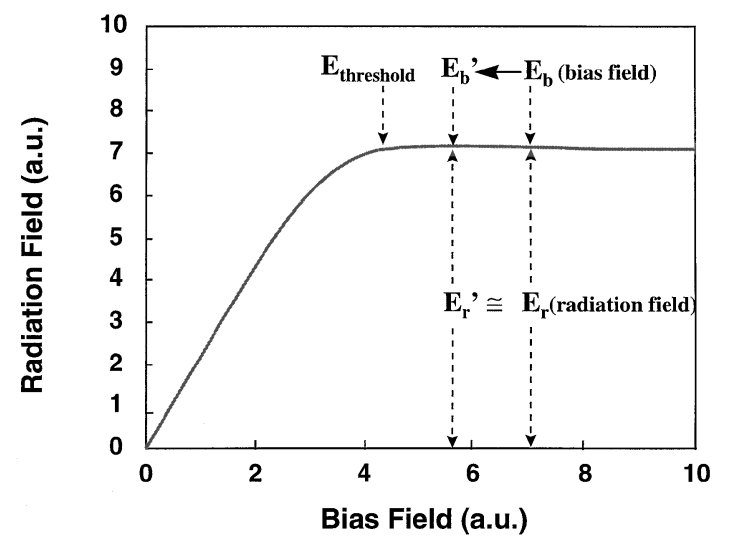

Fig. 3. Typical nonlinear behavior of the radiation power versus the bias field for the III-V photoconductive antenna. The shift of the actual bias from $E_{b}$ to $E_{b^{\prime}}$ owing to other arriving EM pulses will cause only insignificant disturbance since $E_{r^{\prime}}$ (radiation field) is nearly equal to $E_{r}$. Thus the coupling among the photoconductive antenna elements is minimal.

\section{RF Power vs. Angle}

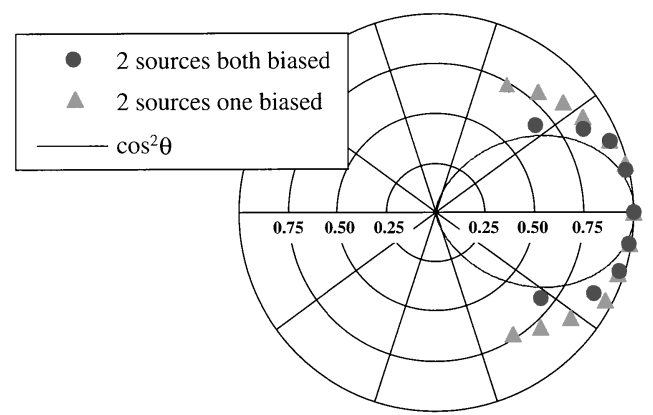

Fig. 4. Polar plot of the EM radiation pattern produced by a single-beam dual-wavelength laser source. Two patterns are scaled to have the same boresight gain. EM radiation beam-width narrowing was observed. The $\cos ^{2} \theta$ plot is shown for comparison. 
It is important to understand that the power that might be realized from laser-induced pulsed picosecond EM source devices is relatively independent of the laser power, provided that there is sufficient fluence to ensure that all the illuminated semiconductor volume is saturated. We have estimated ${ }^{5}$ individual peak powers of $1 \mathrm{~kW}$ (a conversion of the static electric energy, $\varepsilon E^{2} / 2$, stored in a $10-\mathrm{cm}^{2}$ photoconducting antenna area with a typical $1-\mu \mathrm{m}$ optical absorption depth for a 50-ps pulse). From our measurements of radiated field strength, at a distance of $2 \mathrm{~m}$ it was clear that the field was much less than the $1-\mathrm{kV}$ estimate. This finding can be attributed to the fact that the dc-electrode configuration was not optimized and that the laser power was insufficient in these initial trails to achieve saturation fluence. Also, the total radiated power should increase with the square of the number elements.

In summary, we have demonstrated for what is believed to be the first time that two photoconductive antenna elements can be optically excited in an inline serial configuration with a single laser source. The array elements are controlled and reconfigured by optical wavelength multiplexing. We reduced mutual couplings or cross talk among the elements by setting the bias fields at the plateau voltages for the individual semiconductor sources. The radiation beam directionality as well as its intensity can be manipulated by use of a fiber-optic network and choice of input optical powers.

The authors are grateful for helpful discussions with J. B. Thaxter of the Rome Laboratory and with A. J. Devaney and E. A. Morengo of Northeastern
University. Support for E. E. Crisman was provided by the U.S. Air Force Office of Scientific Research and the Department of Physics of Brown University. J. S. Derov's e-mail address is derov@maxwell.rl.plh.af.mil.

*Present address, Department of Physics, Box 1843, Brown University, Providence, Rhode Island 02912.

\section{References}

1. R. A. Gilbert and G. T. Pirrung, presented at the Sixth Annual ARPA Symposium on Photonic Systems for Antenna Applications, Monterey, Calif., March 4-7, 1996.

2. H. Zmuda and E. N. Toughlian, Photonic Aspects of Modern Radar (Artech House, Norwood, Mass., 1994).

3. Ch. Fattinger and D. Grischkowsky, Appl. Phys. Lett. 54, 490 (1989).

4. X.-C. Zhang and D. H. Auston, Appl. Phys. Lett. 59, 768 (1991).

5. D. W. Liu, J. B. Thaxter, and D. F. Bliss, Opt. Lett. 20, 1544 (1995).

6. D. Liu, D. Charette, M. Bergeron, H. Karwacki, S. Adams, and B. Lanning, IEEE Photon. Technol. Lett. 10, 716 (1998).

7. D. W. Liu, P. H. Carr, and J. B. Thaxter, IEEE Photon. Technol. Lett. 8, 815 (1996).

8. E. Marengo, A. J. Devaney, and E. Heyman, IEEE Trans. Antennas Propag. 45, 1098 (1997).

9. E. Marengo, A. J. Devaney, and E. Heyman, IEEE Trans. Antennas Propag. 46, 243 (1997).

10. S. M. Sze, Physics of Semiconductor Devices, 2nd ed. (Wiley, New York, 1981), pp. 638-651.

11. G. M. Wysin, D. L. Smith, and A. Redondo, Phys. Rev. B 38, 12514 (1988).

12. J. T. Darrow, X.-C. Zhang, D. H. Auston, and J. D. Morse, IEEE J. Quantum Electron. 28, 1607 (1992). 